\title{
Discussion
}

\section{Alzheimer Research Forum Live Discussion: Sports Concussions, Dementia, and ApoE Genotyping: What Can Scientists Tell the Public? What's Up for Research?}

\author{
(http://www.alzforum.org/res/for/journal/transcript.asp?liveID=169)
}

Participants: J. Lucy Boyd (Boyd Intellectual Properties), Ira Casson (Long Island Jewish Medical Center), Steve DeKosky (University of Pittsburgh), Hank Feuer (Indiana University), Sam Gandy (Mount Sinai School of Medicine), Zaven Khachaturian (Lou Ruvo Institute), June Kinoshita (Alzheimer Research Forum), Vincent Marchesi (Yale University), Ann McKee (Boston University), Christopher Nowinski (Sports Legacy Institute), Daniel Perl (Mount Sinai School of Medicine), Dushyant Purohit (Mount Sinai), Norman Relkin (Weill Cornell Medical Center), Mark A. Smith (Case Western Reserve University), Gabrielle Strobel (Alzheimer Research Forum), Junming Wang (University of Mississippi Medical Center), Henrik Zetterberg (Sahlgrenska University).

Note: Transcript has been edited for clarity and accuracy.

Zaven Khachaturian: Gabrielle, this is an incredibly important topic not only by itself but also for a better understanding of what is happening in $\mathrm{AD}$ in general.

Gabrielle Strobel: Do you think that concussions/head trauma contribute significantly to $\mathrm{AD}$ in the general population, not just athletes?

Zaven Khachaturian: Probably not directly, but the general idea of disrupting or damaging the microvessels or blood-brain barrier may play a significant role in being an initial triggering factor. Organizing a largescale, population-based longitudinal study will allow us to answer your question and develop new hypotheses about what is going on.

Sam Gandy: I think that to a great extent, we are preaching to the choir. Most or all of us here (I think) believe that there is a significant issue that is largely overlooked. In my mind, the question is how to gauge the extent of chronic traumatic encephalopathy (CTE). Traumatic brain injury (TBI) has received much more attention than CTE. I think that we all agree that the extent of the problem needs some systematic definition. For example, what is the minimal injury that can produce late sequelae?

Gabrielle Strobel: We saw two separate areas: public health questions and research questions. The first issue we were going to discuss is, Do the scientific data about risk from repeated concussions translate into a public health message? Can we state this message simply? All members of the panel, what do you think?

Daniel Perl: Until we know the scope of the problem, especially the minimum degree of trauma leading to 
further sequelae (plus can one episode of head trauma do this?), I do not think we can make any specific public health pronouncements.

Christopher Nowinski: As the person on the panel who has likely suffered the most concussions, I think we are reaching the point where there is a clear public health message - multiple concussions dramatically increase your risk of late-onset neurological sequelae. The public health recommendation in my mind is less clear scientifically. Is it, do not get many concussions? Is it, rest long enough after your concussions? Is it, do not participate in head trauma sports for more than $\mathrm{X}$ number of years?

Sam Gandy: Gabrielle, I do not think that we have a rigorous enough database to make much public comment beyond the extremes. However, even the extremes (dementia pugilistica, Chris Benoit, etc.) deserve greater visibility. And the problem will grow as Iraqi vets return with CTE and post-traumatic stress disorder (PTSD). I agree with Chris that even what is blatantly obvious to most of us would be unknown to most people.

Zaven Khachaturian: Gabrielle, the public health and research questions you had posted in the discussion backgrounder are all very good ones and should be the topic of discussion. Unfortunately, I do not see how we can address these questions without data. Thus, we need to focus on how to organize and fund such a large-scale study knowing that no single agency alone will be able or interested in funding such a mega-study.

Sam Gandy: Zaven, I am not even sure that "a" study will have sufficient scope, which is why I wondered whether your Alzheimer's Disease Research Center (ADRC) model might pertain.

Steven DeKosky: A major issue for smaller injuries will be ascertainment. Minor injuries are not recalled (by their nature), and in sports it is not in the interest of the individual or team to report them. While smaller injuries do surely have effects, remember that the "bar" above which risk of AD can be identified as a risk factor for head trauma is unconsciousness lasting greater than half an hour. A proposal to study this in the population (for more minor injuries) will have to include a very good ascertainment measure...

Sam Gandy: Steve, there are certainly challenges to be overcome, but do you agree with expanding the scale of the inquiry?
Steven DeKosky: Absolutely.

Sam Gandy: Steve, do you like the ADRC model? The cluster of clinical, pathological, and outreach cores coupled with fundamental research would seem appropriate to this problem. Or would the Department of Defense (DoD) be more responsive?

Steven DeKosky: DoD is a good idea; they have great interest in both very mild and severe TBI. This would be a different model than repetitive injury in sport, of course.

Zaven Khachaturian: Sam, I agree that no single study will be adequate to answer all the questions. Yes, the ADRC model, but organized differently and funded differently will be an approach. Namely, you would have to organize a number of core facilities, e.g., biomarker, neuropathological, imaging, clinical neurological, genetic, proteomic, etc. These core facilities, unlike ADRCs, do not need to be in a single location. Essentially I am proposing a virtual center model with an executive committee to coordinate the study.

Vincent Marchesi: All, has anyone measured amyloid- $\beta$ ( $\mathrm{A} \beta)$ autoantibodies in people with posttraumatic injury who also have an apolipoprotein E4 (ApoE4) genotype?

Sam Gandy: Vincent, not that I have seen.

Norman Relkin: Vincent, no, I do not believe that has been done to date.

Gabrielle Strobel: Vincent, Tony Wyss-Coray is working on $\mathrm{A} \beta$ autoantibodies these days.

Vincent Marchesi: If anyone has such serum samples, I would like to make those measurements. I have found that AD patients with ApoE4/4 have elevated anti-A $\beta$ antibodies that were assayed with a peptide fragment of $\mathrm{A} \beta$ derived from the juxtamembrane region of $\mathrm{A} \beta \mathrm{PP}$.

Gabrielle Strobel: Has a reasonably comprehensive study ever been done on retired NFL players, for example? That would seem to be straightforward.

Steven DeKosky: I had proposed such a study to the National Football League (NFL) Hall of Fame. They did not respond. The issue was one of what would be expanded disability claims, I believe. 
Gabrielle Strobel: I believe we have a participant here who has published with the NFL. Hank Feuer, would you like to comment on this issue?

J. Lucy Boyd: There is also a possibility that age makes a difference: that the same injury to a 13-yearold will have a different long-term effect than one received by a 30 -year-old.

Christopher Nowinski: Gabrielle, there have been retrospective surveys done with more than 2,500 NFL players with over three years' experience by the Center for the Study of Retired Athletes at the University of North Carolina (CSRA at UNC) - Kevin Guskiewicz and Julian Bailes.

Sam Gandy: Chris, would you elaborate?

Gabrielle Strobel: Chris, are these published?

Christopher Nowinski: Sam, certainly - CSRA at UNC independently surveyed the health of retired NFL players (neurological status was included) and found that those who remembered having three or more concussions had triple the rate of depression and selfreported memory impairment, about 20 percent, versus those who remembered zero. While the "number" of concussions they remember may not be perfect, those who sustained more of them appeared to be in worse shape. But those are the best large population data out there $[1,2]$.

Steven DeKosky: Right, those are very informative data from the CSRA study. The group that had me involved wanted to study the "later life" risk-specifically in people 20-30 years out because of the risk of what we know to be two separate later complications: $\mathrm{AD}$ and CTE.

Norman Relkin: I think the ascertainment issue that Steve brought up before is quite important relative to choosing the right populations to study.

Sam Gandy: Dan Perl is going to enter via my keyboard. Dan? I think such studies need to include neuropathology. Also, automobile accidents are another huge setting for survival with major head trauma. Another group to examine.

Gabrielle Strobel: All, Henrik is with Kaj Blennow, a leading Swedish group in cerebral spinal fluid (CSF) biomarkers. Henrik, I understand you are doing CSF biomarker work in soldiers. Can you tell us what you found?

Henrik Zetterberg: One question with regard to posttraumatic stress disorders has been that high-impact blows from firing heavy weapons might cause brain damage. In a standardized setting we did not find any such evidence using the same sensitive markers [neurofilament light protein, tau, glial fibrillary acidic protein (GFAP), etc.] as we did when we discovered the very clear evidence of acute axonal injury in post-fight amateur boxers.

Gabrielle Strobel: Henrik, was that impact just blowback from firing weapons or impact sustained in war? And did you measure CSF $\mathrm{A} \beta$ in the soldiers, boxers, soccer players?

Henrik Zetterberg: Gabrielle, the impact was from firing heavy weapons in an experimental setting, not a war situation.

June Kinoshita: It seems that if the concern is about public health implications of head injury in amateur sport, particularly among younger people, one would want to collaborate with children's hospitals about tracking concussion in young athletes.

Henrik Zetterberg: I believe some sports, such as amateur boxing, are advertised as healthy and good both for physical and social training. The results on the pronounced increases in axonal proteins in CSF after mild head blows (e.g., in amateur boxing with head gear and softer gloves) call for caution with allowing children to perform such sports.

Steven DeKosky: One thing that is clear from the wealth of ideas is that different types of studies, requiring different resources and skills, are necessary. For example, following up people in late life is a different design than tracking young athletes. This would not be a one-size-fits-all project, but could be a series of specifically targeted desired datasets.

Christopher Nowinski: Sam, later-life risk is the data needed. The NFL just funded a plan, called the 88 Plan, to pay for care for any former NFL athlete with dementia, regardless of the source. Once the program was publicized, I believe media reports indicated the NFL were quite surprised by the number of families 
that reached out and by the young age of some of them. That list exists somewhere...

Norman Relkin: We have looked at NFL players before retirement, and active boxers. While frank dementia is not expected, relative cognitive impairments and other neurologic findings can be detected, particularly in ApoE4 carriers.

Daniel Perl: We are likely talking about a 20- or more year latency period. The childhood head trauma cases will not likely show anything until much later in life.

Steven DeKosky: I agree with Dan. The reason the NFL Hall of Fame group had raised the question was because they had "follow-up" observing people every year at Canton. When people retired from the NFL they would not have the same kinds of gatherings where they would be repeatedly seen.

June Kinoshita: That is true about the latency between childhood head trauma and later consequences, but if you are talking about intervention, or prevention, it might be a good idea to try to understand possible mechanisms.

Gabrielle Strobel: Steve, would it not be straightforward to identify retired NFL players, I mean players who retired 10, 20 years ago, and invite them into an independently funded study without active involvement from the NFL? After all, they are retired.

Steven DeKosky: That is what I proposed to the Hall of Fame (HoF) committee several years ago. They did not respond. Of course, at that time I thought they would want to support the investigation, and I now understand the reasons that it was not something they were prepared to do because of the liability/cost issues. Now that the NFL has responded with the 88 Plan, it may be a better time to do that.

Christopher Nowinski: Gabrielle, that would be possible. There are unaffiliated retired athlete groups available to discuss.

Norman Relkin: Gabrielle, studying active players prospectively would be a very worthwhile endeavor, and they are very easy to locate.

Sam Gandy: My concern is that we will never get organized sports on board. That we have to back into that area via something less politically and financially charged. Maybe veterans and DoD? Or the motor vehicle accident victims like Dan suggests. Steve, what encouragement did you receive from the NFL? Curiously, the rugby association is actually giving out grants to look at $\mathrm{CTE} / \mathrm{TBI}$ in ruggers.

Steven DeKosky: I was not in contact with the NFL. The NFL Hall of Fame is a separate corporation. They were the ones to whom I responded following the request that came through the Alzheimer's Association. It was coincidental, I think, that the Webster case (and our autopsy study) came to us at the same time. [See http://www.alzforum.org/res/for/journal/detail.asp? liveID=169 for background details on the Webster case.]

Sam Gandy: Steve, where does the NFL HoF project stand? That is new to me.

Steven DeKosky: In the five cases we looked at here and published, the neuropathologist, Ron Hamilton, immediately suspected dementia pugilistica. For me, the experience of seeing no medial temporal tangles but abundant cortical tangles was the major finding.

Norman Relkin: There is a big problem with studying retired players, namely, an ascertainment bias - it is not easy to quantify head trauma from records or recollections.

Sam Gandy: I think that you eventually have to look prospectively to get truly reliable prevalence data.

Norman Relkin: Agreed, Steve, and that is one of several reasons why studying active players prospectively may be more productive.

Steven DeKosky: Norm, I agree but think that both studies should be done. A study of the later-life athletes would help to identify things to study in the prospective group...

Gabrielle Strobel: Sam and Norm, how would you do that? Enroll the highest-exposed people (boxers, perhaps) and follow them?

Norman Relkin: Gabrielle, I would want to draw upon a full range of exposures to permit comparisons, as well as varied ages and genetic backgrounds. 
Sam Gandy: Gabrielle, that would be the general idea. Choice of sport would be important because of the socioeconomic status and other confounds, I think. Not sure whether boxers are "clean" (i.e., of recreational substances) enough, in large numbers. But maybe this bias is not true.

Vincent Marchesi: What are people going to be looking for in living survivors?

Sam Gandy: I think that serial neuropsychological exams and neuroimaging would be the place to start. In other words, an Alzheimer's Disease Neuroimaging Initiative (ADNI) for TBI/CTE.

Christopher Nowinski: It appears we have been joined by Ira Casson, M.D., the Chair of the NFL's Committee on Mild Traumatic Brain Injury, if you have any questions for him. Hi, Ira. Good to see you again.

Gabrielle Strobel: Dr. Casson, what is the most current stance of the NFL on long-term neurologic sequelae after professional football? Is there support for systematic study of long-retired athletes?

Ira Casson: Gabrielle, we are currently performing a study on a large group of retired NFL players.

Gabrielle Strobel: Dr. Casson, is this study conducted by the NFL itself or by independent investigators?

Sam Gandy: Another issue is how many diseases we are talking about, or maybe that is genetically specified. Some dementia pugilistica has amyloid, some is tangle only, yes?

Steven DeKosky: That was going to be my major point. CTE, the classic dementia pugilistica, does not have plaque sufficient to make the call of AD. TBI is also a risk factor for $\mathrm{AD}$ - and these cases look like classic AD. So there are two different entities at autopsy.

Henrik Zetterberg: Steven, the original hypothesis when we designed the amateur boxing study was that boxers might have the relatively $\mathrm{AD}$-specific changes of elevated phospho-tau and low $\mathrm{A} \beta_{42}$ in CSF. However, they did not display these changes at all. Instead, we found pronounced damage to subcortical axons and glia cells.
Ann McKee: Gabrielle and Steve, I think that many cases of late-life CTE are misdiagnosed as AD. Although there are considerable overlaps, they have clear distinctions that can be parsed out. The issue of $\mathrm{A} \beta$ deposition in older age groups always complicates the issue. I also agree with Dan from my own experience with a large brain bank, that a single head injury, such as from a motor vehicle accident, can result in CTE.

Steven DeKosky: Ann, I understand that is a risk. But if you look at what is published, it is not a lot, and comes from very good places.

Daniel Perl: Finding the cases misdiagnosed as AD is not straightforward and has not been done. The key is the distribution of the neurofibrillary tangles (NFTs), and you have to know what you are looking for to find it.

Steven DeKosky: I agree, Norm. You would have to study them in comparison to matched groups to determine prevalence in them.

Ann McKee: Steve and Dan, I agree - you have to look for the differences, and if you do not think to do that, you could miss the distinction.

Steven DeKosky: We have always known that ApoE has two brain "risks": one for decreased clearance of $\mathrm{A} \beta$ and the other the decrease in repair abilities that has been demonstrated in mice. That difference may be what leads to either CTE pathology or facilitates AD. Or it may just be the loss of brain reserve that comes with serious injury.

Junming Wang: Steve, we saw neurogenesis deficits in as early as three-month-old 3xTg mouse hippocampus. I think this lower rate of producing new, healthy cells is an important factor. A biomarker of neural progenitor cell proliferation may help.

Steven DeKosky: Junming, it would help for animal studies but not for human. I was referring to decreased sprouting after injury. Both mechanisms, of course, may be interfering with recovery...

Vincent Marchesi: Does anybody have any comment of the observation of Laskowitz that a 17-amino-acid fragment of ApoE improves the rate of recovery of experimental head injuries $[3,4]$ ? 
J. Lucy Boyd: Would it be possible to assume professional football players of 10 or more years have had more head trauma than the general population? That would solve the ascertainment issue.

Vincent Marchesi: What are people going to be looking for?

Norman Relkin: J. Lucy Boyd, that is a reasonable assumption, but would still not permit assessment of differences in cumulative exposure. Several studies have failed to find such a correlation.

Sam Gandy: Dan and I are agreeing here that defining minimal exposure and maximal latency would be key parameters to define before initiating a study with any chance of being close to comprehensive. Agreed, anyone?

Gabrielle Strobel: Our next agenda question was, Assuming we agree on the minimal public health message Chris spelled out, then how broad is it beyond professional football? Wrestling? Soccer? Hockey? College? High school sports? Do we have data to say anything beyond a general message of concern?

Christopher Nowinski: New splinter question - when do we believe athletes are most likely laying the groundwork for long-term progressive brain damage? Is it even through all years of sports? More risk when trauma is received ages five-10, 10-20, 20+? Any thoughts? I am curious.

Sam Gandy: Great question, Chris. I have no clue.

J. Lucy Boyd: Chris, I am very curious about that also.

Gabrielle Strobel: Sam, panel, is the age and latency question where ApoE comes in? Less capacity to repair damage over time?

Sam Gandy: I wish that I could say that I knew anything for sure. The sample sizes are too small to be sure. Dan is commenting here that ApoE genotype may specify how quickly cognition declines, i.e., like the age at onset in AD. Here it would be modulating latency.

Steven DeKosky: Chris's question is a key one. Just as we believe AD "emerges" on the background of an aging brain, it may be that the injuries are present but not clinically detectable until combined with agerelated change. .

Sam Gandy: Steve, one thing that might speak to this issue is that some mid-life risks for AD can persist into old age despite resolution of the risk factor per se. For example, mid-life obesity that remains a risk later even if the obesity is gone.

Norman Relkin: Chris, one very interesting window on the question you ask is the phenomenon of sudden death from massive brain edema after serial concussions close in time. Called Second Impact syndrome, this is unfortunately seen in young $(<20$-year-old) athletes but rarely seen in older ones. Clearly there are differences in how the brain reacts to trauma as a function of age.

Sam Gandy: Norm, the catastrophic edema reaction extends to children, maybe babies.

Norman Relkin: In terms of public health messages, Second Impact syndrome is a good example of a posttraumatic neurologic syndrome that is relatively poorly publicized and catastrophic when it occurs.

Sam Gandy: Second Impact, latency are both issues that we all recognize but not even the general medical population is aware of.

Gabrielle Strobel: All, in light of latency of disease process and age, does sitting out more games, or resting a week or two instead of three days, make a difference for the athlete?

Christopher Nowinski: Gabrielle, I just returned from a UCLA conference with Dave Hovda and Chris Giza, and their groups' studies in the rat model consistently seem to show that rats who do not "rest" after brain trauma, physical and/or cognitively, have more physical brain damage/neuronal dropout. So longer rest after concussion also should theoretically have a major impact on long-term sequelae.

Daniel Perl: There are no human data I am aware of that resting in between multiple concussions is protective against the neurodegenerative sequelae. It may be protective against malignant cerebral edema but not the late stuff.

Christopher Nowinski: Regarding the public health message on sports, I think we also have to agree first 
whether it is "concussions" alone or cumulative trauma that is responsible for CTE. Is it safer to get knocked out once every five years or take 10,000 sub-concussive blows to the head in the same time but have zero concussions? When we have that answered, we will have a better idea of which sports are most dangerous for the brain. Any thoughts?

Steven DeKosky: Chris's question is the excellent reason to do the prospective study carefully...

Mark A. Smith: Regarding Chris's question, are there any data from animal studies looking at repetitive lowlevel injury?

Ira Casson: The evidence from boxing indicates that CTE is related to length of career and number of bouts, not the number of times the boxer was knocked out.

Gabrielle Strobel: That would argue for cumulative effect of sub-threshold impact, not just full concussions. Any data like that from the NFL study?

Ira Casson: Gabrielle, we are looking at this as part of our study. John Trojanowski's group worked with a group in China and reported a study of the effects of a number of relatively low-level head impacts on a group of genetically identical laboratory rats (a few years ago).

Norman Relkin: Gabrielle, a substantial component of a boxer's exposure to head trauma comes from sparring rather than actual bouts.

Sam Gandy: Practice injuries in football too, Norm.

Daniel Perl: In the UNC study there were nearly as many concussions during practice as during games.

Mark A. Smith: Having played soccer (30+ years) and rugby (10 years), this is clearly a question of selfinterest.

Steven DeKosky: Norm, any information from autopsies? Any suggestion of factors that would lead to AD or to CTE at pathology?

Norman Relkin: Good, question, Steve, but there are too few autopsy series to answer that.

Gabrielle Strobel: How about a genetic study looking for frequency of genes implicated in AD and PD in re- tired athletes and correlating that with their neurologic health? Doable? Too complex?

Sam Gandy: Gabrielle, I think that that might be an eventual goal, but I am not sure we are ready until we know how many clinical and neuropathological "bins" there are. We could certainly look at the "usual suspects," though.

J. Lucy Boyd: I believe that 100 years from now, the understanding will be that the human brain is fragile, more fragile than we want it to be. Just as today we know "do not shake a baby." We do not say, do not shake a baby harder than X, or longer than X, or more times than $X$. In the future, I believe it will become clear (with better imaging, testing, and studies) that all brain trauma is negative and probably creates some long-term effect.

Mark A. Smith: J. Lucy Boyd, I agree. . . the question is whether these are avoidable and/or worth it on an individual or societal level. In other words, are the rewards (often huge) worth the risks (even if high)?

Henrik Zetterberg: J. Lucy Boyd, a comment on that. We were actually surprised that amateur boxers who took 15 or more hits to their heads (without being knocked out) but felt fine afterwards had neurofilament light protein levels in their CSF similar to what we see after minor brain infarctions. I agree with you completely that the brain is more fragile than we want it to be and particularly susceptible to shaking.

Norman Relkin: Henrik, I thought that was a very compelling finding. Was it not difficult, though, to get athletes to agree to undergo spinal taps?

Henrik Zetterberg: No, it was not. But perhaps that has to do with the fact that we perform spinal taps much more often than in the US For instance, every patient who seeks medical advice for memory problems undergoes a spinal tap to exclude neuroborreliosis and assess AD biomarkers, and if not, he or she (or relatives) might argue that it was a careless physician and ask for a second opinion.

Norman Relkin: Thanks, Henrik. I do think professional athletes might be more reticent out of concern about negative after-effects, but I have not approached any recently to ask. 
Gabrielle Strobel: Henrik, can you teach the US how to adopt that attitude here? Reluctance to do spinal taps, and fear of them, holds back some of the most innovative $\mathrm{AD}$ research here.

Henrik Zetterberg: Gabrielle, it is just to be persistent. If people are willing to undergo colonoscopy on a regular basis, which is very likely to be much more troublesome, it should be possible to perform one or two spinal taps.

Gabrielle Strobel: We were going to discuss whether we are ready to recommend ApoE genotyping. Given that Genetic Information Nondiscrimination Act (GINA) does not cover life and long-term care insurance, do I sense consensus that the answer may be "still no"? Panel, all?

Norman Relkin: Gabrielle, I do not think there is sufficient evidence at this time to recommend the use of ApoE genotyping as a marker for risk of neurologic sequelae in athletes.

Sam Gandy: I agree with Norm. I think the issue is how to build the database to say anything concrete. The old "if your child had an E4 allele, would you let him/her play contact sports in high school?" Is it desirable to look for the answer to that question?

Christopher Nowinski: I thought Norm said it well in his video presentation. Too early for all, but will help some. Maybe an easier question is, If your child played contact sports and received a few concussions, would you have him or her tested for E4 and then include that in a decision of whether to retire early? I think that is a yes, and people should be informed of that option. There is nothing worse than finding out after the fact that you should have known better.

Gabrielle Strobel: Chris, your framework makes sense at a gut level. There would have to have been an event already that causes one to worry, rather than just a totally pre-emptive genetic test.

Sam Gandy: But Chris, do you think you have the knowledge base to make that decision or are you just being ultra-cautious? A problem with this message is that it implies that the E4 non-carriers will not dement, and that is the wrong message.

Christopher Nowinski: One of the worst aspects of my retirement experience was discovering that doctors believed I was not smart or mature enough (at 24) to be told that research exists that shows concussions have long-term detrimental consequences, just in case I overreacted. So if the data are the data, then I say tell people and let them make their own choices. And no, I do not believe that telling people ApoE4 increases risks tells others they can be careless. The CTE cases I have been involved with have not involved ApoE4 for the most part. It just takes good education.

Gabrielle Strobel: So Chris, do you see the generally negative stance toward ApoE testing as a kind of paternalism by physicians?

Christopher Nowinski: Gabrielle, thanks for the loaded question. . . The interpretation of my experience was that they did not want to tell me things that would scare me or change my behavior much. While not everyone has the capacity to interpret reams of medical research, and I do not have the experience of being a clinician, I think in some cases you have got to lay the cards on the table and let people choose for themselves.

J. Lucy Boyd: Chris, I agree.

Sam Gandy: The paternalism/condescension thing bugs me, too, Chris. Since Norm has gone, I will suggest that we begin thinking about organizing something along the lines of what he and I did for the Alzheimer's Association ApoE Genotyping Consensus in 1995ish. A praecis was published in The Lancet and longer papers were collated into a volume of Annals NYAS. Zaven has volunteered his journal to play that role, but I think that we might want to get The Lancet on board again up front.

Gabrielle Strobel: I think the time for that would be good, Sam.

Mark A. Smith: Any thoughts on the influence of performance-enhancing drugs, which are likely common in professional (and amateur) sports?

Gabrielle Strobel: On performance-enhancing drugs, I would like to ask Dan and Ann and all neuropathologists here, Can you distinguish a difference between their impact on brain ("steroid brain") and the impact of blows to the head?

Sam Gandy: Gabrielle, that is another confound to define, I think. 
Ann McKee: Although steroids have been shown to augment $\mathrm{A} \beta$ and tau pathology in animal models, I am not aware of any human study specifically demonstrating that chronic steroid use results in anything remotely similar to CTE.

Mark A. Smith: Ann, I was thinking more along the lines of a double whammy - a two-hit type of thing [5].

Gabrielle Strobel: Ann, pathologically speaking, is there such a thing as "steroid brain"? I heard that reference in a CNN story about TBI.

Ann McKee: I have seen the references in the lay press as well, but as far as I know there is a neuropsychological aspect to chronic steroid use but not a neuropathological one characterized by abnormalities of tau, although animal studies suggest that steroid use might potentially accentuate the tau abnormalities.

Dushyant Purohit: To all pathologists here, considering there are few autopsies of TBI in individual centers, a collective brain bank could be a good idea. It will be immediately useful for a study, because clinical cases recruitment and follow-ups would take a much longer time to get any significant results.

Ira Casson: Ann, can you tell us where to find the animal studies that show neuropathological effects of steroids on the brain?

Sam Gandy: Glucocorticoids (GCs) are toxic to hippocampal neurons. Read anything that Bruce McEwen has published over the past 30 years.

Ira Casson: I have discussed this with Bruce McEwen. He told me that no one has studied the relationship between steroids and tau.

Sam Gandy: If that is what Bruce said, I would not challenge. GCs do kill neurons and increase $\mathrm{A} \beta$ generation and accumulation.

Ann McKee: Ira, Frank LaFerla's group published a recent paper [6] showing increased $\mathrm{A} \beta$ and tau with glucosteroids in the triple transgenic animal model of AD. Gabrielle, my take on this issue of CTE is, as mentioned early on, that it is much more prevalent than realized, and that it may also in fact accelerate what we consider to be AD (perhaps along the lines of the double-hit hypothesis). Although Steve mentioned that his CTE cases had no medial temporal neurofibrillary tangles, my experience would be the opposite - that there is considerable medial temporal lobe, especially amygdala, pathology.

Daniel Perl: I agree with Ann (as usual); our cases had very heavy medial temporal pathology, including involvement of amygdala.

Gabrielle Strobel: All, if memory serves, Hank Feuer is a coauthor on NFL papers about return to play by concussed players. Perhaps you have a question? We would love to hear from him.

Hank Feuer: Education and communication have been the hallmarks of my care of the concussed athlete and all those involved.

Gabrielle Strobel: I would like to invite the panelists to make a closing statement. In keeping with the structure of the discussion, perhaps tell us what you think we know with reasonable certainty now and what you would like to study next if money was available.

Steven DeKosky: Here is my concluding statement: just as our discussion today showed what a complex area this is, the manner in which we address it in experimentation and data gathering can also vary. While I need more time to digest the suggestions of information provided, my view at present is that there are several biological questions that I would be very interested in trying to answer. A major one is after a lifetime in which there were several or multiple blows to the head, or even one significant injury, what leads to either chronic traumatic encephalopathy (dementia pugilistica pathology) or classical Alzheimer's disease pathology? They are certainly very different. This would be the reason to study people in late life who have or do not have cognitive impairment but were matched for experience in, for example, professional sports. The other would be the initiation of a prospective study, with public health implications, of younger people playing sports and looking to see what the shortto intermediate-term effects on cognition are. This latter may be more difficult because of reluctance or unwillingness to have such things documented (especially if they are done during sports and might precipitate the loss of a player). These are difficulties known to all of us. But a central discussion might evolve a very good way to do this. I believe the first study I propose would be somewhat easier to initiate. It is not the only way 
to do it. Working with the Department of Defense and the United States Department of Veterans Affairs on subjects who have had head trauma would also provide very useful data.

Variables to be considered in devising studies: severe injury one-time versus multiple smaller injuries, age at which injuries occurred (young children playing sports versus professional football players versus soldiers), and how long after the exposure one would look - shortto intermediate-term, long-term/late life.

In addition to the fact that we will learn something with significant implications for public health, I also think we will answer a fundamental question about how injury affects the brain. I am fascinated by the idea that what appear at this point to be similar injuries may lead to either chronic traumatic encephalopathy or to Alzheimer's disease. One has neurofibrillary tangles in a different distribution than Alzheimer's; the other has tangles and amyloid plaque. What determines which pathology emerges? We have the ability to make progress in a variety of ways here as well as do good things for public health.

Gabrielle Strobel: Last call for comments, all.

Ira Casson: Gabrielle, I always enjoy hearing other perspectives and always keep an open mind. My opinions are always based on solid scientific research.

Gabrielle Strobel: You listened to some of the best scientific minds in the field of Alzheimer's and CTE research today.

Sam Gandy: My final comment is that we do not yet have enough real data to make much of a fuss vis-à-vis public policy, but we should not ignore the problem to the extent that it has been in the past. Operations Iraqi Freedom and Enduring Freedom (OIF/OEF) veterans with TBI certainly represent another population that merits representation at the table.

Gabrielle Strobel: Thank you for all your work on this, Sam.

\section{REFERENCES}

[1] Guskiewicz KM, Marshall SW, Bailes J, McCrea M, Cantu RC, Randolph C, Jordan BD (2005) Association between recurrent concussion and late-life cognitive impairment in retired professional football players. Neurosurgery 57, 719-726.

2] Guskiewicz KM, Marshall SW, Bailes J, McCrea M, Harding HP, Matthews A, Mihalik JR, Cantu RC (2007) Recurrent concussion and risk of depression in retired professional football players. Med Sci Sports Exerc 39, 903-909.

[3] Laskowitz DT, McKenna SE, Song P, Wang H, Durham L, Yeung N, Christensen D, Vitek MP (2007) COG1410, a novel apolipoprotein E-based peptide, improves functional recovery in a murine model of traumatic brain injury. J Neurotrauma $\mathbf{2 4}$, 1093-1107.

[4] Wang H, Durham L, Dawson H, Song P, Warner DS, Sullivan PM, Vitek MP, Laskowitz DT (2007) An apolipoprotein Ebased therapeutic improves outcome and reduces Alzheimer's disease pathology following closed head injury: evidence of pharmacogenomic interaction. Neuroscience 144, 1324-1333.

[5] Zhu X, Raina AK, Perry G, Smith MA (2004) Alzheimer's disease: the two-hit hypothesis. Lancet Neurol 3, 219-226.

[6] Green KN, Billings LM, Roozendaal B, McGaugh JL, LaFerla FM (2006) Glucocorticoids increase amyloid-beta and tau pathology in a mouse model of Alzheimer's disease. J Neurosci 26, 9047-9056. 